\title{
The age-related effect on cognitive performance in cognitively healthy elderly is mainly caused by underlying $A D$ pathology or cerebrovascular lesions: implications for cutoffs regarding cognitive impairment
}

Emma Borland ${ }^{1,2^{*}}$, Erik Stomrud ${ }^{1,3}$, Danielle van Westen ${ }^{1,4}$, Oskar Hansson ${ }^{1,3}$ and Sebastian Palmqvist ${ }^{1,3^{*}}$

\begin{abstract}
Background: As research in treatments for neurocognitive diseases progresses, there is an increasing need to identify cognitive decline in the earliest stages of disease for initiation of treatment in addition to determining the efficacy of treatment. For early identification, accurate cognitive tests cutoff values for cognitive impairment are essential.

Methods: We conducted a study on 297 cognitively healthy elderly people from the BioFINDER study and created subgroups excluding people with signs of underlying neuropathology, i.e., abnormal cerebrospinal fluid [CSF] $\beta$ amyloid or phosphorylated tau, CSF neurofilament light (neurodegeneration), or cerebrovascular pathology. We compared cognitive test results between groups and examined the age effect on cognitive test results.

Results: In our subcohort without any measurable pathology $(n=120)$, participants achieved better test scores and significantly stricter cutoffs for cognitive impairment for almost all the examined tests. The age effect in this subcohort disappeared for all cognitive tests, apart from some attention/executive tests, predominantly explained by the exclusion of cerebrovascular pathology.

Conclusion: Our study illustrates a new approach to establish normative data that could be useful to identify earlier cognitive changes in preclinical dementias. Future studies need to investigate if there is a genuine effect of healthy aging on cognitive tests or if this age effect is a proxy for higher prevalence of preclinical neurodegenerative diseases.

Keywords: Cognitive assessment, True norms, Robust norms, Cutoff, Normative, Age, Preclinical pathology
\end{abstract}

\footnotetext{
*Correspondence: emma.borland@med.lu.se; sebastian.palmqvist@med.lu.se

${ }^{1}$ Clinical Memory Research Unit, Department of Clinical Sciences, Lund University, Malmö, Sweden

Full list of author information is available at the end of the article
}

(c) The Author(s). 2020 Open Access This article is licensed under a Creative Commons Attribution 4.0 International License, which permits use, sharing, adaptation, distribution and reproduction in any medium or format, as long as you give appropriate credit to the original author(s) and the source, provide a link to the Creative Commons licence, and indicate if changes were made. The images or other third party material in this article are included in the article's Creative Commons licence, unless indicated otherwise in a credit line to the material. If material is not included in the article's Creative Commons licence and your intended use is not permitted by statutory regulation or exceeds the permitted use, you will need to obtain permission directly from the copyright holder. To view a copy of this licence, visit http://creativecommons.org/licenses/by/4.0/ The Creative Commons Public Domain Dedication waiver (http://creativecommons.org/publicdomain/zero/1.0/) applies to the data made available in this article, unless otherwise stated in a credit line to the data. 


\section{Background}

Cognitive test norms are used for comparing a person's performance to a large group of individuals of the same age, education, and gender. Norms based on persons without underlying cerebral pathology are crucial for identification of cognitive decline in neurocognitive disorders at early stages. To accurately capture the earliest declines in progression, it is necessary to know at what cognitive performance a cognitive decline should be suspected. It is preferable to capture an individual's actual changes in cognitive level; however, when there are no available longitudinal data, reference data from cognitively healthy individuals can be used for comparison. A mild decline in cognitive function can be caused by underlying pathology, such as beta-amyloid [1, 2], neurofibrillary tangles, cerebral infarctions, and Lewy bodies [3], and it is well known that pathophysiologic processes of neurocognitive disorders begin many years before a diagnosis of dementia $[4,5]$. Research on neurocognitive disorders, such as Alzheimer disease (AD), has therefore switched focus from diagnosing clinically symptomatic $\mathrm{AD}$, to a biological definition of the disease, in order to identify preclinical individuals with only subtle cognitive decline for earlier recognition and intervention $[5,6]$.

Traditionally, test norms were established from subjectively cognitively healthy people, which later was improved by creating robust test norms using longitudinal data from people without clinical progression in cognitive symptoms [7-11]. Further, cognitive test scores have been stratified by age, as test results are correlated with age in cognitively healthy persons [12]. These previous methods did not account for the presence of relevant cerebral pathologies and might thus have a lower sensitivity for identifying subtle cognitive changes that accompany preclinical dementias.

In this study, we hypothesized that (1) excluding cognitively healthy controls with underlying measurable in vivo brain pathologies, would result in improved test results and more strict cutoffs for cognitive impairment, and (2) the effect of age on cognitive test results to a large extent is driven by individuals with preclinical neurodegenerative disease, and by excluding these from normative studies, the effect of age would be reduced. To examine this, we compared test results from cognitively healthy people based on the earlier methods explained above, with test results from people without certain brain pathologies, including abnormal accumulation of beta-amyloid $(\mathrm{A} \beta$ ) and elevated phosphorylated tau (Ptau) as a marker for Alzheimer pathology (measured using cerebrospinal fluid [CSF] $\mathrm{A} \beta 42 / 40$ and P-tau), cerebrovascular lesions (measured using magnetic resonance imaging [MRI]), or axonal injury (measured using CSF neurofilament light (NfL)). We established cognitive test norms and cutoffs for cognitive impairment from cognitively healthy elderly persons without these underlying pathologies and compared these to cutoffs in traditionally classified cognitively healthy controls. We also investigated the effect of age, years of education, and gender on cognitive tests in groups with and without underlying measurable in vivo pathologies.

\section{Methods \\ Participants}

Cognitively healthy elderly persons were included from the prospective Swedish BioFINDER Study (http://biofinder.se), and participants for this study were enrolled between July 6, 2009, to March 4, 2015. The population was consecutively included from the large population-based Malmö Diet and Cancer Study [13]. The inclusion criteria for being included as a healthy control in BioFINDER were (1) a score on MMSE $\geq 28$ points at the screening visit, (2) age $\geq 60$ years old, and (3) fluent in the Swedish language. The exclusion criteria were (1) presence of subjective cognitive impairment, (2) presence of significant neurological disease (e.g., stroke, Parkinson disease, multiple sclerosis), (3) significant unstable systemic illness or organ failure that makes it difficult to participate in the study, (4) severe psychiatric disease (e.g., severe depression or psychotic syndrome), (5) dementia or mild cognitive impairment (MCI), and (6) current significant alcohol or substance misuse. Eligible participants were evaluated by physicians wellexperienced in dementia disorders regarding cognitive status, fulfillment of inclusion criteria and absence of exclusion criteria in a 1 -h-long interview including a semi-structured interview for Clinical Dementia Rating (CDR) [14] scoring. Only subjects with a complete data set of CDR at baseline and at least one follow-up visit (either a 2- or 4-year follow-up), available CSF ratio of $\mathrm{A} \beta 42 / 40$, CSF P-tau, CSF NfL, assessment of white matter lesions (WML), and cortical infarctions were included. This resulted in a study population of 297 participants. In a subanalysis, we also applied different test cutoffs on both the controls and patients with subjective cognitive decline (SCD), collectively termed cognitively unimpaired [5] $(n=$ 529) from BioFINDER. The study criteria for the SCD cohort have been described elsewhere [15].

\section{Procedures \\ Cognitive tests}

Eight cognitive tests were examined in the present study, covering the cognitive domains of executive function, attention, episodic, and semantic memory as well as visuospatial function. The Alzheimer's Disease Assessment Scale (ADAS) is an instrument designed specifically to evaluate the severity of cognitive and noncognitive 
behavioral dysfunctions characteristic of people with AD. In this study, we used ADAS Naming Objects and Fingers (here ADAS naming), where the subject is assessed in their ability to name different objects and fingers, and ADAS 10-word delayed recall (here ADASdelayed recall), where the participant was previously exposed to 10 words during three learning trials, and memory function is tested for delayed recall. For both ADAS subscales, points are counted as words the subject does not acknowledge or recall, meaning higher points equals worse test results [16]. Animal Fluency is a semantic verbal fluency test where the participant is asked to produce as many words in the category "animals" as possible in $60 \mathrm{~s}$. This tests for cognitive flexibility when shifting between animal categories (e.g., horse-cowsheep and dog-cat-mouse) related to frontal functioning, and clustering related to temporal lobe disturbance [17]. In this study, we scored the number of animals the individual produces. A Quick Test of Cognitive Speed Color and Form (AQT) consists of 40 figures with different colors and forms, assessing cognitive processing speed and task-switching. The time it takes to name the color and form for all figures equals the test score [18]. Stroop color and Word Test (here Stroop) assesses the ability to inhibit the cognitive interference that occurs when the processing of one stimulus affects the simultaneous processing of another stimulus [19]. The participant reads words printed in another color, leading to a prefrontal cortex activation [20]. The time it takes to complete the test becomes the test score. Trail Making Test A and B (TMT A and B) provide information on visual search, speed of processing, mental flexibility, and executive functions [21]. In TMT A, the participant draws lines between 25 numbers sequentially, and in TMT B participants alternate between sequential numbers and letters (i.e., 1-A, 2-B, 3-C etc.). The time it takes to complete the test equals the test score [21]. For Symbol Digit Modalities Test (SDMT), the participant uses a reference key to pair specific symbols with numbers, receiving one point for every correct answer within the response time of $90 \mathrm{~s}$. The test assesses divided attention, visual scanning, tracking, and motor speed [20].

\section{MRI}

All participants were examined using the same 3-T MRI scanner [22]. White matter lesions and cortical infarctions were assessed by visual inspection for all the participants. White matter lesions were graded using the 4point Fazekas scale (0-3) on T2 FLAIR images, and participants with a Fazekas score $\geq 2$ were defined as having clinically significant cerebrovascular pathology [23]. Presence of supra- or infracortical infarctions were assessed on 2D FLAIR and T1-weighted MPPRAGE images. Liquidated tissue with or without surrounding gliosis was regarded as infarction and included if $\geq 2 \mathrm{~mm}$ in size.

\section{CDR assessment}

Eligible participants were thoroughly assessed at the Memory Clinic, Skåne University Hospital, at baseline and at least a 2- or 4-year follow-up by a physician well experienced in dementia disorders. This assessment included a semi-structured CDR interview. CDR is a numeric scale scoring $0-3$ points used to quantify the severity of symptoms of dementia based on measures of memory, orientation, judgment and problem solving, community affairs, home and hobbies and personal care [14].

\section{CSF analysis}

The procedure and analysis of CSF followed the Alzheimer's Association Flow chart for CSF biomarkers [15, 24]. CSF $\mathrm{A} \beta 42, \mathrm{~A} \beta 40$, and P-tau were analyzed using Elecsys immunoassays on all participants. CSF NfL was analyzed with ELISAs (NF-light ${ }^{\circ}$ ELISA kit; UmanDiagnostics AB, Umeå, Sweden) as previously described [25].

\section{Creating different normative samples}

The participants in our study were all included in the sample cohort (A) of 297 people (see under "Participants"). Four different subgroups (B, C, D, and E) were created based on different exclusion criteria. In cohort $\mathrm{B}$, we excluded persons with clinically progressive cognitive decline according to CDR during $\geq 2$ years. For all participants, CDR assessments were conducted at baseline and at a 2-year follow-up except for two participants with missing data at 2-year follow-up for whom data from the 4-year visit was used; these two participants had not progressed in CDR. Clinical progression was defined as a CDR sum of boxes $\geq 0.5$, corresponding to the traditional method of establishing robust test norms [7-10]. In cohort $\mathrm{C}$, we excluded persons with any AD pathology, here defined as either abnormal $A \beta$ or tau. Abnormality was defined using previously established cut-offs. For $A \beta$, it was a CSF $A \beta 42 / 40$ ratio of $<0.059$ [26] and for tau a CSF P-tau level $\geq 28 \mathrm{pg} / \mathrm{mL}$ [27]. In cohort $\mathrm{D}$, we excluded persons with cerebrovascular pathology (white-matter lesions or infarctions), defined by a Fazekas score $\geq 2$ in any brain region and/or any visual cortical infarctions on MRI scans. In cohort $\mathrm{E}$, we excluded people with any underlying measurable in vivo pathology; abnormal CSF A $\beta$ or tau pathology, cerebrovascular pathology or CSF NfL, a marker of neurodegeneration that is affected also in frontotemporal dementia [28] and atypical Parkinson diseases [29] or simply a measure of neurodegeneration $[5,30]$. The CSF NfL cutoff for cohort $\mathrm{E}$ was created with mixture modeling statistics [31] on cognitively healthy controls and patients with subjective cognitive decline or mild cognitive impairment in BioFINDER $(n=823)$. CSF NfL- 
levels were logarithmized because of skew distribution and several outliers, and the cutoff was defined as log NfL $>3.33 \mathrm{pg} / \mathrm{mL}$.

\section{Statistical analysis}

Mean scores and standard deviations were established for tests of executive function (TMT A and B, SDMT, Stroop, and Animal Fluency), attention (AQT), episodic memory (ADAS-delayed recall), and semantic memory (ADAS naming). Correlation coefficients for age and test results were calculated using Spearman correlation. To investigate if the absence of significant correlations for most cognitive tests was caused by a lack of statistical power, we conducted bootstrap analysis with 500 bootstraps from 100 individuals (to ensure bootstrapping of the smallest cohort (cohort E) would not result in finding significance when gaining a larger cohort and more statistical power) and calculated mean correlation values as well as mean $p$ values. Association between years of education and test results were conducted with Spearman correlation and association between gender and test results with Mann-Whitney. The association between groups and cognitive test results controlled for age, education and sex were calculated using multivariable linear regression with test score as outcome and disease pathology as a predictor $(0=$ present or $1=$ absent $)$. Cutoff values for cognitive impairment for the cognitive tests were calculated by adding/subtracting 1.5 standard deviations (SD) to/from the mean values, e.g., for SDMT where the aim is to achieve as many points as possible, 1.5 SD is subtracted from the mean score to calculate the cutoff value, whereas for TMT, 1.5 SD was added to mean scores. Two-sided $p$ value of $<0.05$ indicated statistical significance. The above analyses were all calculated with SPSS Statistics version 25. Confidence intervals for the cutoffs and correlation coefficients were estimated from 500 bootstrap samples using R Version 3.5.2.

\section{Results}

\section{Demographics}

The total study population (cohort A) consisted of 297 persons between the ages of 64-88 years old, mean age 73.5 years (see Table 1 for demographics).

\section{Correlation between age and cognitive test results}

In the total population (cohort A) and the cohort without amyloid or tau pathology (cohort C), there was a significant association between age and all other cognitive test results. In cohort B (no clinical progression in CDR score), there was no significant association between age and ADAS-delayed recall or ADAS naming. In the cohort excluding only cerebrovascular pathology (cohort D), there was no significant correlation between age and

Table 1 Demographics

\begin{tabular}{|c|c|c|c|c|c|}
\hline & A. Study cohort & $\begin{array}{l}\text { B. No progress in } \\
\text { CDR }\end{array}$ & $\begin{array}{l}\text { C. No amyloid or tau } \\
\text { pathology }\end{array}$ & $\begin{array}{l}\text { D. No vascular } \\
\text { pathology }\end{array}$ & $\begin{array}{l}\text { E. No measurable in } \\
\text { vivo pathology }\end{array}$ \\
\hline Participants, N & 297 & 278 & 223 & 161 & 120 \\
\hline MMSE score, mean $(S D)^{a}$ & $29.1(0.9)$ & $29.1(0.9)$ & $29.1(0.9)$ & $29.1(0.9)$ & $29.1(0.9)$ \\
\hline Age, mean (SD) & $73.5(5.0)$ & $73.4(5.0)$ & $73.4(5.0)$ & $72.5(4.6)$ & $72.2(4.6)$ \\
\hline Education years, mean (SD) & $12.3(3.7)$ & $12.3(3.8)$ & $12.2(3.6)$ & $12.5(0.9)$ & $12.6(3.5)$ \\
\hline \multicolumn{6}{|l|}{ Gender } \\
\hline Male & $39.4 \%$ & $38.8 \%$ & $39.5 \%$ & $41.6 \%$ & $42.5 \%$ \\
\hline Female & $60.6 \%$ & $61.2 \%$ & $60.5 \%$ & $58.4 \%$ & $57.5 \%$ \\
\hline APOE $\varepsilon 4$ ( $\geq 1$ allele) & $27.3 \%$ & $25.9 \%$ & $16.3 \%$ & $30.8 \%$ & $20.3 \%$ \\
\hline \multicolumn{6}{|c|}{ Prevalence of abnormal biomarkers } \\
\hline CSF A $342 / 40<0.059$ & $24.9 \%$ & $23.0 \%$ & $0 \%$ & $24.2 \%$ & $0 \%$ \\
\hline CSF P-tau $>28 \mathrm{pg} / \mathrm{mL}$ & $15.2 \%$ & $13.3 \%$ & $0 \%$ & $14.3 \%$ & $0 \%$ \\
\hline Log CSF NfL > 3.33 pg/mL & $2.4 \%$ & $1.8 \%$ & $2.2 \%$ & $1.2 \%$ & $0 \%$ \\
\hline White matter lesions ${ }^{\mathrm{b}}$ & $45.5 \%$ & $43.9 \%$ & $44.8 \%$ & $0 \%$ & $0 \%$ \\
\hline$\geq 1$ Cortical infarctions & $1.3 \%$ & $1.1 \%$ & $1.7 \%$ & $0 \%$ & $0 \%$ \\
\hline \multicolumn{6}{|l|}{ Comorbidity } \\
\hline Hypertension & $43.4 \%$ & $42.8 \%$ & $43.5 \%$ & $41.0 \%$ & $43.3 \%$ \\
\hline Diabetes & $10.1 \%$ & $10.4 \%$ & $9.4 \%$ & $11.2 \%$ & $10.0 \%$ \\
\hline Ischemic heart disease & $6.7 \%$ & $6.8 \%$ & $6.3 \%$ & $4.3 \%$ & $2.5 \%$ \\
\hline
\end{tabular}

${ }^{\mathrm{a}}$ All groups have a high MMSE score due to the inclusion criteria of MMSE $\geq 28$ points. ${ }^{\mathrm{b}}$ Measured as Fazekas score $\geq 2$ point in any region 
test results for ADAS-delayed recall, ADAS naming, Animal Fluency, or AQT, but there was for Stroop, TMT A and B, and SDMT (i.e., a remaining age effect for tests of attention and executive function). In the cohort without any measurable pathology (cohort E), the age effect only remained for TMT A and TMT B (Table 2). The age effect remained for difference scores (TMT-B minus TMT-A) for all cohorts (Additional table 1). To examine if the absence of significant correlations for most cognitive tests was caused by a lack of statistical power $(n=297$ in cohort A, $n=120$ in cohort E), we performed further sensitivity analyses. We created 500 bootstrap samples from cohorts A and E, respectively, with 100 participants in each sample. The mean $p$ values for Spearman correlations in the 500 bootstrap samples from cohort A (with only $n=100$ ) still indicated significant correlations between age and all cognitive tests as in the original analysis. For cohort E, there were only significant correlations for age and TMT A and B in the bootstrap analysis, indicating that the difference in age effect on cognitive test results between cohort $\mathrm{A}$ and $\mathrm{E}$ was not caused by a difference in statistical power (Additional table 2).

The association between cognitive test results, gender, and education

Education correlated with test results for all tests apart from ADAS-delayed recall, ADAS naming, and TMT A in cohort A, where higher education was associated with improved test scores. In cohort $\mathrm{E}$, there was an education effect for ADAS-delayed recall, Stroop, TMT B, and SDMT (Additional table 3). We found a significant association between female gender and ADAS-delayed recall in cohorts $\mathrm{A}, \mathrm{B}$, and $\mathrm{C}$, and between male gender and improved test scores in AQT for cohort D. No significant differences were found between men and women for any other cognitive tests regardless of cohort (A-E) (Additional table 4).

\section{Establishing cutoffs for cognitive impairment}

In Table 3, we present mean test scores of each cognitive test for all five cohorts. The cutoff for cognitive impairment was defined as the mean value $\pm 1.5 \mathrm{SD}$ (depending on whether higher score equaled worse or better performance) [32, 33]. In cohort E, all cutoffs for cognitive impairment differed significantly (were stricter) from the total population (cohort A) apart from ADAS naming. Cutoffs in cohort $\mathrm{E}$ differed significantly from the traditional method for establishing robust norms (cohort B) for Animal Fluency, TMT A, TMT B, and SDMT (Table 4). In Fig. 1, we present percent differences in cutoffs (at $\pm 1.5 \mathrm{SD}$ from mean) between the total cohort (cohort A) and the cohort without pathology (cohort E). Of those cutoffs that differed significantly, the cutoff was 6.2 to $19.9 \%$ stricter in cohort E. All test results showed an improvement in cognitive test score when excluding those with underlying pathologies. The effect of the underlying pathology on the cognitive test result was independent of age, gender, and education (Additional table 5). We also investigated the sensitivity for detecting

Table 2 Correlation coefficients for test results and age in each cohort

\begin{tabular}{|c|c|c|c|c|c|}
\hline Cognitive Test & $\begin{array}{l}\text { A. Study } \\
\text { Cohort } \\
(n=297)\end{array}$ & $\begin{array}{l}\text { B. No Progress } \\
\text { in CDR } \\
(\mathrm{n}=278)\end{array}$ & $\begin{array}{l}\text { C. No Amyloid } \\
\text { or Tau } \\
\text { Pathology } \\
(\mathrm{n}=223)\end{array}$ & $\begin{array}{l}\text { D. No } \\
\text { Vascular } \\
\text { Pathology } \\
(\mathrm{n}=161)\end{array}$ & \begin{tabular}{|l} 
E. No \\
measurable in- \\
vivo pathology \\
$(n=120)$
\end{tabular} \\
\hline $\begin{array}{l}\text { ADAS-delayed } \\
\text { recall }\end{array}$ & $0.143^{*}$ & 0.116 & $0.160^{*}$ & 0.029 & -0.008 \\
\hline ADAS-naming & $0.142^{*}$ & 0.112 & $0.135^{*}$ & 0.107 & 0.044 \\
\hline Animal Fluency & $-0.193 * * *$ & $-0.144^{*}$ & $-0.147^{*}$ & 0.024 & 0.126 \\
\hline AQT & $0.224 * * *$ & $0.203^{* * *}$ & $0.223^{* * *}$ & 0.016 & -0.010 \\
\hline Stroop & $0.368^{* * *}$ & $0.373^{* * *}$ & $0.386^{* * *}$ & $0.189^{*}$ & 0.129 \\
\hline TMT A & $0.337^{* * *}$ & $0.331^{* * *}$ & $0.365^{* * *}$ & $0.289^{* * *}$ & $0.289^{* *}$ \\
\hline TMT B & $0.387^{* * *}$ & $0.375^{* * *}$ & $0.406^{* * *}$ & $0.344 * * *$ & $0.340 * * *$ \\
\hline SDMT & $-0.419^{* * *}$ & $-0.413^{* * *}$ & $-0.371^{* * *}$ & $-0.253 * *$ & -0.132 \\
\hline
\end{tabular}

Correlation coefficients for age and test results conducted with Spearman correlation. Only significant correlation coefficients are colored. Yellow boxes for coefficients $\geq 0.1$ to $<0.2$, orange boxes for $\geq 0.2$ to $<0.3$, red boxes for $\geq 0.3$. ${ }^{*}$ Correlation is significant at the 0.05 level, ${ }^{* *}$ correlation is significant at the 0.01 level ${ }^{* * *}$ correlation is significant at the 0.001 level 
Table 3 Cognitive test results for each cohort

\begin{tabular}{llllll}
\hline Cognitive test & A. Study cohort & $\begin{array}{l}\text { B. No progress } \\
\text { in CDR }\end{array}$ & $\begin{array}{l}\text { C. No amyloid or } \\
\text { tau pathology }\end{array}$ & $\begin{array}{l}\text { D. No vascular } \\
\text { pathology }\end{array}$ & $\begin{array}{l}\text { E. No measurable in } \\
\text { vivo pathology }\end{array}$ \\
\hline ADAS-delayed recall & $1.98(1.93)$ & $1.79(1.72)$ & $1.80(1.78)$ & $1.81(1.80)$ & $1.59(1.56)$ \\
ADAS naming & $0.38(0.79)$ & $0.34(0.74)$ & $0.36(0.79)$ & $0.30(0.75)$ & $0.24(0.68)$ \\
Animal Fluency & $21.7(5.5)$ & $22.1(5.4)$ & $22.0(5.5)$ & $22.4(5.3)$ & $23.0(5.3)$ \\
AQT & $66.0(12.9)$ & $65.1(12.3)$ & $65.8(12.9)$ & $63.8(12.0)$ & $63.3(11.1)$ \\
Stroop & $28.9(7.4)$ & $28.5(7.0)$ & $28.6(7.5)$ & $27.5(6.9)$ & $26.9(6.7)$ \\
TMT A & $46.0(17.0)$ & $45.6(17.1)$ & $45.6(16.9)$ & $43.0(14.8)$ & $41.0(12.0)$ \\
TMT B & $104.4(50.8)$ & $101.8(49.4)$ & $101.9(49.0)$ & $97.1(44.7)$ & $90.0(36.5)$ \\
SDMT & $37.0(8.4)$ & $37.5(8.3)$ & $37.5(8.5)$ & $38.4(8.3)$ & $39.1(8.0)$
\end{tabular}

Data are shown as mean (SD). A: The entire population. B: No progress in Clinical Dementia Rating (CDR) over 2 years. C: No preclinical AD (i.e., CSF Aß42/40 and P-tau not abnormal). D: No vascular pathology. E: No measurable pathology (i.e., no AD pathology, cerebrovascular pathology or increased NfL)

preclinical pathology using the cutoffs from cohort $\mathrm{E}$ (without underlying pathology) compared to cutoffs from cohort A (the total population) in cognitively unimpaired individuals in BioFINDER (controls and patients with subjective cognitive decline, $N=528$ ). Overall, we found that using cutoffs from group $\mathrm{E}$ increased the sensitivity for detecting preclinical $\mathrm{AD}$ or cerebrovascular pathology and also improved the overall performance (higher Youden index), compared to using cutoffs from group A (Additional tables 6-7).

\section{Discussion}

In this study, we examined cognitive test scores, cutoffs for cognitive impairment, and age effects in 297 cognitively healthy elderly people with and without certain brain pathologies, including cerebrovascular pathology on MRI, CSF A $342 / 40$, and P-tau as markers for preclinical AD and CSF $\mathrm{NfL}$ as a marker of neurodegeneration. The significant, agerelated effect on cognitive test results disappeared for cognitive tests when excluding people with underlying preclinical pathology (the effect was predominantly caused by the exclusion of cerebrovascular pathology) for all tests, except for TMT A and TMT B that test attention/executive function (Table 2). This somewhat fits with a previous paper showing that age-related decline to a large extent can be explained by underlying brain markers such as cortical thickness, different regional brain volumes, structural connectivity, and white matter hyperintensities [34], suggesting age itself does not affect cognition but is a confounder for the association between underlying brain markers or disease mechanisms and cognitive test results.

When excluding the cognitively healthy elderly with underlying pathologies, we found significantly stricter cutoff values for cognitive impairment for all the examined cognitive tests, apart from ADAS naming, compared to the total cohort of cognitively healthy elderly (Fig. 1, Table 4). The improvement in cognitive test score when excluding underlying pathologies was independent of age, gender, and education (Additional table 5). To our knowledge, this is the first study that illustrates the effect of producing test norms from certified cognitively normal individuals without underlying AD pathology or cerebrovascular pathology.

In the total population (cohort A) and the cohort without amyloid or tau pathology (cohort C), we found a significant correlation between age and all test results,

Table 4 Cognitive test cutoffs at 1.5 SD from mean for cohorts A, B and E

\begin{tabular}{|c|c|c|c|}
\hline & Cohort A cutoffs (95\% Cl) & Cohort B cutoffs (95\% Cl) & Cohort E cutoffs (95\% Cl) \\
\hline ADAS-delayed recall & $4.88(4.59-5.16)$ & $4.38(4.13-4.61)$ & $3.93(3.63-4.20)$ \\
\hline ADAS naming & $1.57(1.42-1.71)$ & $1.44(1.29-1.58)$ & $1.25(1.01-1.48)$ \\
\hline Animal Fluency & $13.5(13.1-13.9)$ & $14.0(13.6-14.4)$ & $15.0(14.4-15.7)$ \\
\hline $\mathrm{AQT}$ & $85.3(83.6-86.9)$ & $83.5(81.8-85.2)$ & $80.0(77.8-82.1)$ \\
\hline Stroop & $40.0(39.0-40.9)$ & $39.0(38.0-39.8)$ & $36.9(35.1-38.6)$ \\
\hline TMT A & $71.5(69.2-73.9)$ & $71.3(68.9-73.8)$ & $59.0(56.5-61.2)$ \\
\hline TMT B & $180.6(172.1-189.0)$ & $176.0(167.8-184.4)$ & 144.6 (135.5-152.5) \\
\hline SDMT & $24.3(23.7-25.0)$ & $25.1(24.4-25.8)$ & $27.2(26.1-28.3)$ \\
\hline
\end{tabular}

Cutoffs (1.5 SD from mean) created from 500 bootstrap samples. We found significantly improved cutoffs in cohort E compared to cohort A for all tests apart from ADAS naming. We found significantly improved cutoffs in cohort $E$ compared to the traditional method of creating robust norms (cohort B) for Animal Fluency, TMT A, TMT B, and SDMT, i.e., non-overlapping $95 \%$ Cls (presented in bold) 


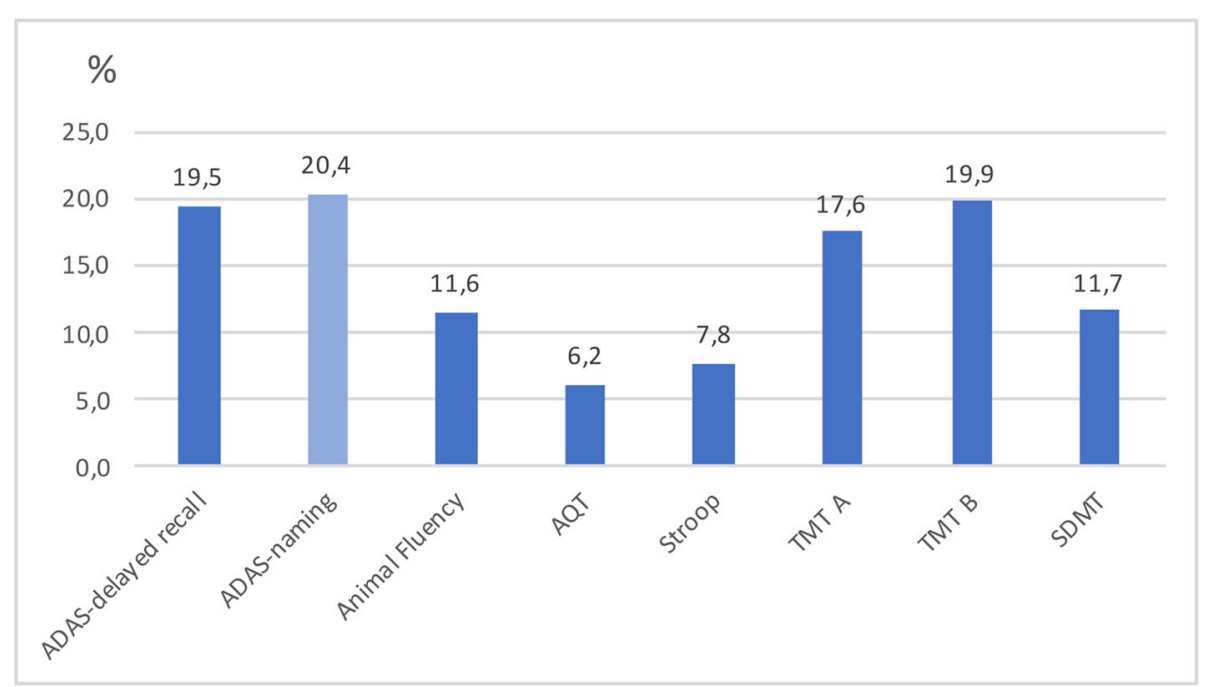

Fig. 1 Percent change in test cutoffs (1.5 SD from mean) between the total population and those without measurable brain pathologies. All test cutoffs were significantly stricter in cohort $\mathrm{E}$ (no measurable pathologies) compared to cohort A (whole population) except for ADAS naming (Table 4). Percent changes were calculated by dividing cutoffs for cohort E with cutoffs for cohort A

which is congruent with the common notion that aging is accompanied by cognitive decline [35] and that most cognitive test results are affected by age [2,36], regardless of level of amyloid [37]. There are however previous studies showing more prominent effects of preclinical AD (i.e., $\mathrm{AD}$ pathology in cognitively healthy controls) on cognition $[2,38,39]$. There could be several causes for why we do not find similar results in our study. Firstly, when examining the effect of underlying pathologies on test results (Additional table 5) on AD-negative participants, we are only comparing with a small number of participants with AD pathology $(N=74)$, i.e., there could be a lack of statistical power. Secondly, we use CSF measures of amyloid pathology instead of amyloid-PET as in other previous studies, which is more closely related to cognition [38, 40]. Differences in results could also be cutoff dependent. Nonetheless, we also want to point out that (supporting the present findings) there are several large studies showing that the effect of AD pathology is not always apparent at baseline, but instead becomes apparent after longitudinal follow-ups of around 4 years (as shown in, e.g., the BioFINDER, AiBL, and ADNI studies [41, 42]).

For cohort B, there were no age effects for ADAS delayed recall or ADAS naming. When excluding people with cerebrovascular pathology (cohort D), the age effect on cognitive test scores disappeared for ADAS-delayed recall, ADAS naming, Animal Fluency, and AQT, besides, was reduced for the remaining tests. In our cohort without measurable pathology (E), we found similar results for age effect on cognitive tests as in cohort D, though the age effect disappears even for Stroop and SDMT. However, the above suggests vascular pathology stands for most of the age effect.
For the cognitive tests TMT A and TMT B, a significant age correlation remained even when excluding people with underlying pathologies. This suggests either that there is an actual cognitive decline related to a seemingly healthy aging process or that the age-related decline is caused by an unmeasurable pathology or cause. The latter case is a limitation of this study and also of the current research field in general. Unaccounted pathologies that could cause cognitive decline, such as TDP-43 [43] in, e.g., Limbic-predominant age-related TDP-43 encephalopathy (LATE) [44] or frontotemporal dementias and alpha-synuclein in parkinsonian disorders, are not yet possible to measure in vivo and could therefore not be excluded from our study. Other possible causes of the remaining age-related decline in test results which are not accounted for are difficulties in psychomotor speed, gait speed, or decline in visual competence. However, these abilities are included in other cognitive tests where the age effect disappeared, speaking against them being the cause of the remaining age effect. Speed variables are frequently found to have moderate to large relations with age across adulthood; however there are many types of measures of speed in cognitive testing [45]. Different types of speed are tested in TMT A (motor speed) and B (motor speed and internal responses) [46], SDMT (processing speed as well as motor speed), Stroop (processing speed), and Animal Fluency (mental speed) [20], suggesting decrease in motor speed is the main cause of remaining age-related decrease in TMT $\mathrm{A}$ and $\mathrm{B}$. To test the age effect on internal responses in our study, we calculated the difference score (TMT-B minus TMT-A) for each cohort. Mean difference scores in cohort A was $57.9 \mathrm{~s}$ and in cohort E $48.4 \mathrm{~s}$, suggesting 
time for internal responses decreases in cohort E. Analyses, however, showed that the age effect for the difference score remained for all cohorts (see Additional table 1). Previous studies have however suggested the relationship between TMT A and B is more complicated than only differing in motor speed, as TMT B has a longer distance and visual scanning is more difficult [47]. In another perspective, it has previously been suggested that the degree of slowing in speed is greater for tasks involving spatial information than for those involving verbal information [48], which could suit with our findings that the age effect still remains for TMT A and B but disappears for others testing speed such as Animal Fluency, AQT, and Stroop, all including verbal information.

Our findings that cutoffs change when excluding patients with preclinical pathology confirms the findings from the two previous studies that excluded underlying AD pathology $[2,39]$. Cutoffs for cohort $\mathrm{E}$ were also stricter than the traditional method for establishing robust norms (cohort B) in Animal Fluency, TMT A, TMT B, and SDMT (Table 4). We also found reduced SD for all tests in cohort E compared to cohorts A and B (Table 3), implicating the variance in test scores was smaller for cohort $\mathrm{E}$ and, thus, that it was a more homogeneous group than the total population. We do not, however, believe that the present study was less stringent in clinically ruling out cognitive impairment, since previously published normative scores are more in line with those from group A (total population) than the ones from cohort $\mathrm{E}$ (without underlying pathology) $[17,20,49,50]$.

To our knowledge, no other study has excluded cognitively healthy participants based on biomarkers of vascular, amyloid, P-tau, and neurodegenerative abnormalities to investigate the effect on test norms and the relationship with age. However, Hassenstab et. al. investigated test norms on patients without positive biomarkers for preclinical AD (T-tau, P-tau, and A $\beta 42 / 40$ ), as well as abnormal hippocampal volume [39]. As in our study, they assessed patients with TMT A, TMT B, and Animal Fluency. They found slightly improved cutoffs (mean + $1.5 \mathrm{SD}$ ) for TMT A at $46.7 \mathrm{~s}$ and $118.7 \mathrm{~s}$ for TMT B for a younger $(<75$ years) group, however slightly worse for persons $\geq 75$ years of age (TMT A of $52.2 \mathrm{~s}$ and TMT B $148.8 \mathrm{~s}$ ) closer to cohort E's cutoffs of TMT A $59.0 \mathrm{~s}$ and $144.6 \mathrm{~s}$ for TMT B. For Animal Fluency however, they found less strict cutoffs for both younger and older subjects, 13.4 vs. 11.2 words, compared to our cutoff at 15.0 words. In their study, variance in cognitive performance attributable to age remained for TMT A, TMT B, and Animal Fluency in their group without preclinical AD, however, was less profound than in the preclinical group. Another previous study investigated test results on persons without positive biomarkers for $A \beta$ for people aged >40 years. Means of cognitive tests including TMT A and B were calculated. Cutoffs $($ mean + 1.5 SD) for $\mathrm{A} \beta$-negative individuals were $51.3 \mathrm{~s}$ for TMT A and $123.8 \mathrm{~s}$ for TMT B, lower than cohort E's cutoffs of TMT A $59.0 \mathrm{~s}$ and $144.6 \mathrm{~s}$ for TMT B, but could be explained by their young cohort [2].

In terms of implementing stricter cutoffs such as those from group E, there is a potential risk of overdiagnosing cognitive impairment in a clinical setting. They are therefore probably not always suitable in, e.g., a primary care setting. However, in order to screen for preclinical disease such as in the enrolment process of clinical AD trials or a tentative future scenario where disease-modifying $\mathrm{AD}$ treatments are available, such new and more sensitive cutoffs could be suitable to use as a first screening step before applying biomarkers to verify any potential underlying pathology. This potential is hinted at in our analysis when using the cutoffs for detecting preclinical $\mathrm{AD}$ and cerebrovascular lesions in the cognitive unimpaired participants in BioFINDER (Additional tables 6-7). But as our cutoffs are produced from a small cohort of only 120 participants, we do not suggest they are the definite cutoffs to be used for this purpose (nor that these tests necessarily are the most suitable). Instead, we believe this study illustrates the change in cutoff values when removing underlying pathologies and, most importantly, the findings problematize the concept of normal cognitive aging and the previously used method of stratifying cutoffs according to age. For future normative studies, we suggest that in addition to excluding individuals with subjective cognitive impairment, dementia, MCI, alcohol or substance misuse, or any other condition prone to cause cognitive impairment as usually is the case for control cohorts, we also propose that one considers excluding subjects with underlying cerebrovascular pathology, AD pathology, or signs of neurodegeneration. To simplify the establishment of such test norms from large population-based materials, it may be possible to use plasma biomarkers instead of invasive lumbar punctures or PET scans to screen for AD pathology (e.g., plasma P-tau that performs similar to CSF P-tau [51] or the combination of plasma P-tau181 and plasma A $\beta 42$ / $40[52,53]$ ) and axonal/cerebrovascular lesions (e.g., plasma NfL [54, 55]).

An advantage with this study is our consecutively included cohort of individuals assessed with CSF biomarkers, structural neuroimaging, and cognitive assessments including longitudinal CDR. A weakness of our study is the relatively small group without any pathology (120 people). However, we showed that the difference between the total study cohort (A) and those without any pathology (E) regarding the age effect on cognition was not caused by a difference in statistical power (Additional table 2). Further, we chose to not include MRI measures of atrophy, partly because of the uncertainty of which brain regions to measure, and 
partly because brain atrophy is a downstream marker, i.e., possibly caused by old age and not just neurocognitive diseases. Instead, we chose to investigate CSF NfL as a measure of axonal damage and neurodegeneration, as it can identify preclinical diseases for which we have no other specific diagnostic biomarker, such as atypical Parkinson disease and diseases in the frontotemporal lobe disease spectrum as well as act as a general marker for neurodegeneration [5, 28-30].

\section{Conclusions}

In summary, we propose that most age-related decline in cognitive tests is caused by underlying pathological mechanisms and that the traditionally used age stratification might have been caused by a higher proportion of preclinical neurocognitive disorders in the older age groups. Stratifying norms according to age could result in a delayed identification of early cognitive decline in early stages of neurocognitive diseases, especially due to cerebrovascular pathology. Further research with a larger population is necessary to confirm our findings that the age effect disappears for most cognitive tests in people without underlying pathology.

\section{Supplementary information}

Supplementary information accompanies this paper at https://doi.org/10. 1186/s13195-020-00592-8.

Additional file 1: Table S1. Correlation coefficients for difference scores in Trail Making Test measures with age in each cohort.

Additional file 2: Table S2. Mean correlation coefficients and $p$-values between test results and age from bootstrap analysis.

Additional file 3: Table S3. Correlation between years of education and test results.

Additional file 4: Table S4. Associations between gender and cognitive test scores.

Additional file 5: Table S5. Linear regression models examining the effect of underlying pathologies on test results.

Additional file 6: Table S6. Comparison between cutoffs from cohort $A$ and $E$ for detecting preclinical AD in cognitively unimpaired BioFINDER participants.

Additional file 7: Table S7. Comparison between cutoffs from cohort $A$ and $E$ for detecting preclinical cerebrovascular disease in cognitively unimpaired BioFINDER participants.

Additional file 8: Table S8. Cutoffs (+/- 1.5 SD from mean) for all cognitive tests in different groups.

\section{Acknowledgements}

Not applicable.

\section{Funding}

Work at the authors' research center was supported by the European Research Council, the Swedish Research Council, the Knut and Alice Wallenberg foundation, the Marianne and Marcus Wallenberg foundation, the Strategic Research Area MultiPark (Multidisciplinary Research in Parkinson's disease) at Lund University, the Swedish Alzheimer Foundation, the Swedish Brain Foundation, The Parkinson foundation of Sweden, The Parkinson Research Foundation, the Skåne University Hospital Foundation, and the Swedish federal government under the ALF agreement. Open access funding provided by Lund University.
Availability of data and materials

The study data are not publicly available for download, but might be retrieved from the principal investigator professor Oskar Hansson.

\section{Authors' contributions}

EB analyzed and interpreted the data regarding measurable pathologies and test results. ES assessed patients with cognitive tests and clinical dementia rating (CDR). DvW analyzed MRI scans of participants. $\mathrm{OH}$ is head of the research group. SP supervised the first author. ES, DVW, OH and SP revised the manuscript draft. The authors read and approved the final manuscript.

\section{Ethics approval and consent to participate}

The study was approved by the regional ethical committee at Lund University, Lund, Sweden. All participants gave their written informed consent to participate in the study.

\section{Consent for publication}

Not applicable.

\section{Competing interests}

$\mathrm{EB}, \mathrm{ES}, \mathrm{DW}$, and SP declare that they have no competing interests. $\mathrm{OH}$ has acquired research support (for the institution) from Roche, GE Healthcare, Biogen, AVID Radiopharmaceuticals and Euroimmun. In the past 2 years, he has received consultancy/speaker fees (paid to the institution) from Biogen and Roche.

\section{Author details}

${ }^{1}$ Clinical Memory Research Unit, Department of Clinical Sciences, Lund University, Malmö, Sweden. ²Department of Neurology, Skåne University Hospital, Malmö, Sweden. ${ }^{3}$ Memory Clinic, Skåne University Hospital, Malmö, Sweden. ${ }^{4}$ Department of Neuroradiology, Lund University, Lund, Sweden.

Received: 15 November 2019 Accepted: 2 March 2020

Published online: 24 March 2020

\section{References}

1. Jansen WJ, Ossenkoppele R, Tijms BM, et al. Association of cerebral amyloidbeta aggregation with cognitive functioning in persons without dementia. JAMA Psychiatry. 2018;75:84-95.

2. Bos I, Vos SJB, Jansen WJ, et al. Amyloid-beta, tau, and cognition in cognitively normal older individuals: examining the necessity to adjust for biomarker status in normative data. Front Aging Neurosci. 2018;10:193.

3. Wilson RS, Leurgans SE, Boyle PA, Schneider JA, Bennett DA. Neurodegenerative basis of age-related cognitive decline. Neurology. 2010; 75:1070-8.

4. Buckley RF, Mormino EC, Amariglio RE, et al. Sex, amyloid, and APOE epsilon4 and risk of cognitive decline in preclinical Alzheimer's disease: findings from three well-characterized cohorts. Alzheimers Dement. 2018;14: 1193-203.

5. Jack CR Jr, Bennett DA, Blennow K, et al. NIA-AA research framework: toward a biological definition of Alzheimer's disease. Alzheimers Dement. 2018;14:535-62.

6. Dubois B, Hampel H, Feldman HH, et al. Preclinical Alzheimer's disease: definition, natural history, and diagnostic criteria. Alzheimers Dement. 2016; 12:292-323.

7. De Santi S, Pirraglia E, Barr W, et al. Robust and conventional neuropsychological norms: diagnosis and prediction of age-related cognitive decline. Neuropsychology. 2008:22:469-84.

8. Grober E, Mowrey W, Katz M, Derby C, Lipton RB. Conventional and robust norming in identifying preclinical dementia. J Clin Exp Neuropsychol. 2015; 37:1098-106.

9. Harrington $K D$, Lim $Y Y, A m e s ~ D$, et al. Using robust normative data to investigate the neuropsychology of cognitive aging. Arch Clin Neuropsychol. 2017;32:142-54.

10. Holtzer R, Goldin Y, Zimmerman M, Katz M, Buschke H, Lipton RB. Robust norms for selected neuropsychological tests in older adults. Arch Clin Neuropsychol. 2008;23:531-41.

11. Sliwinski M, Lipton RB, Buschke $H$, Stewart W. The effects of preclinical dementia on estimates of normal cognitive functioning in aging. J Gerontol B Psychol Sci Soc Sci. 1996;51:P217-25. 
12. Lezak MD. Neuropsychological assessment. 3rd ed. New York: Oxford University Press; 1995.

13. Manjer J, Carlsson S, Elmstahl S, et al. The Malmo Diet and Cancer Study: representativity, cancer incidence and mortality in participants and nonparticipants. Eur J Cancer Prev. 2001;10:489-99.

14. Hughes $C P$, Berg L, Danziger $W L$, Coben LA, Martin RL. A new clinical scale for the staging of dementia. Br J Psychiatry. 1982;140:566-72.

15. Palmqvist $\mathrm{S}$, Zetterberg $\mathrm{H}$, Blennow $\mathrm{K}$, et al. Accuracy of brain amyloid detection in clinical practice using cerebrospinal fluid beta-amyloid 42: a cross-validation study against amyloid positron emission tomography. JAMA Neurol. 2014;71:1282-9.

16. Rosen WG, Mohs RC, Davis KL. A new rating scale for Alzheimer's disease. Am J Psychiatry. 1984;141:1356-64.

17. Troyer AK, Leach L, Strauss E. Aging and response inhibition: normative data for the Victoria Stroop test. Neuropsychol Dev Cogn B Aging Neuropsychol Cogn. 2006;13:20-35

18. Nielsen NP, Wiig EH, Warkentin S, Minthon L. Clinical utility of color-form naming in Alzheimer's disease: preliminary evidence. Percept Mot Skills. 2004;99:1201-4.

19. Van der Elst W, Van Boxtel MP, Van Breukelen GJ, Jolles J. The Stroop colorword test: influence of age, sex, and education; and normative data for a large sample across the adult age range. Assessment. 2006;13:62-79.

20. Strauss E, Sherman EMS, Spreen O. A compendium of neuropsychological tests: administration, norms, and commentary. 3rd ed. New York: Oxford University Press; 2006.

21. Tombaugh TN. Trail Making Test A and B: normative data stratified by age and education. Arch Clin Neuropsychol. 2004;19:203-14.

22. Palmqvist S, Schöll M, Strandberg O, Mattsson N, Stomrud E, Zetterberg $H$ Blennow K, Landau S, Jagust W, Hansson O. Earliest accumulation of $\beta$ amyloid occurs within the default-mode network and concurrently affects brain connectivity. Nature communications. 2017;8(1):1214.

23. Wahlund LO, Westman E, van Westen D, et al. Imaging biomarkers of dementia: recommended visual rating scales with teaching cases. Insights Imaging. 2017;8:79-90

24. Blennow K, Hampel H, Weiner M, Zetterberg H. Cerebrospinal fluid and plasma biomarkers in Alzheimer disease. Nat Rev Neurol. 2010;6:131-44.

25. Norgren N, Rosengren L, Stigbrand T. Elevated neurofilament levels in neurological diseases. Brain Res. 2003:987:25-31.

26. Palmqvist S, Janelidze S, Stomrud E, et al. Performance of fully automated plasma assays as screening tests for Alzheimer disease-related beta-amyloid status. JAMA Neurol. 2019;76(9):1060-9.

27. Blennow K, Shaw LM, Stomrud E, et al. Predicting clinical decline and conversion to Alzheimer's disease or dementia using novel Elecsys Abeta(142), pTau and tTau CSF immunoassays. Sci Rep. 2019;9:19024.

28. Meeter LH, Dopper EG, Jiskoot LC, et al. Neurofilament light chain: a biomarker for genetic frontotemporal dementia. Ann Clin Transl Neurol. 2016;3:623-36

29. Sako W, Murakami N, Izumi Y, Kaji R. Neurofilament light chain level in cerebrospinal fluid can differentiate Parkinson's disease from atypical parkinsonism: evidence from a meta-analysis. J Neurol Sci. 2015;352:84-7.

30. Olsson B, Portelius E, Cullen NC, et al. Association of cerebrospinal fluid neurofilament light protein levels with cognition in patients with dementia, motor neuron disease, and movement disorders. JAMA Neurol. 2018;76(3): 318-25

31. Benaglia T, Chauveau D, Hunter DR, Young D. Mixtools: an R package for analyzing finite mixture models. J Stat Softw. 2009;32:1-29.

32. Petersen RC, Smith GE, Waring SC, Ivnik RJ, Tangalos EG, Kokmen E. Mild cognitive impairment: clinical characterization and outcome. Arch Neurol. 1999:56:303-8

33. Jak AJ, Bondi MW, Delano-Wood L, et al. Quantification of five neuropsychological approaches to defining mild cognitive impairment. Am J Geriatr Psychiatry. 2009;17:368-75.

34. Hedden T, Schultz AP, Rieckmann A, et al. Multiple brain markers are linked to age-related variation in cognition. Cereb Cortex. 2016;26:1388-400.

35. Blazer DG, Wallace RB. Cognitive aging: what every geriatric psychiatrist should know. Am J Geriatr Psychiatry. 2016;24:776-81.

36. Salthouse TA. Selective review of cognitive aging. J Int Neuropsychol Soc. 2010;16:754-60.

37. Oh H, Madison C, Haight TJ, Markley C, Jagust WJ. Effects of age and betaamyloid on cognitive changes in normal elderly people. Neurobiol Aging. 2012;33:2746-55.
38. Harrington KD, Schembri A, Lim YY, et al. Estimates of age-related memory decline are inflated by unrecognized Alzheimer's disease. Neurobiol Aging. 2018;70:170-9.

39. Hassenstab J, Chasse R, Grabow P, et al. Certified normal: Alzheimer's disease biomarkers and normative estimates of cognitive functioning. Neurobiol Aging. 2016:43:23-33.

40. Palmqvist S, Mattsson N, Hansson O. Alzheimer's disease neuroimaging I. Cerebrospinal fluid analysis detects cerebral amyloid-beta accumulation earlier than positron emission tomography. Brain. 2016;139:1226-36.

41. Insel PS, Weiner M, Mackin RS, et al. Determining clinically meaningful decline in preclinical Alzheimer disease. Neurology. 2019:93:e322-33.

42. Donohue MC, Sperling RA, Petersen R, et al. Association between elevated brain amyloid and subsequent cognitive decline among cognitively normal persons. JAMA. 2017;317:2305-16.

43. Wilson RS, Yu L, Trojanowski JQ, et al. TDP-43 pathology, cognitive decline, and dementia in old age. JAMA Neurol. 2013;70:1418-24.

44. Nelson PT, Dickson DW, Trojanowski JQ, et al. Limbic-predominant agerelated TDP-43 encephalopathy (LATE): consensus working group report Brain. 2019;142:1503-27.

45. Salthouse TA. Aging and measures of processing speed. Biol Psychol. 2000; 54:35-54.

46. Llinas-Regla J, Vilalta-Franch J, Lopez-Pousa S, Calvo-Perxas L, Torrents Rodas D, Garre-Olmo J. The Trail Making Test. Assessment. 2017;24:183-96.

47. Gaudino EA, Geisler MW, Squires NK. Construct validity in the Trail Making Test: what makes part B harder? J Clin Exp Neuropsychol. 1995;17:529-35.

48. Tomer A, Cunningham WR. The structure of cognitive speed measures in old and young adults. Multivariate Behav Res. 1993;28:1-24.

49. Nogueira J, Freitas S, Duro D, Almeida J, Santana I. Validation study of the Alzheimer's disease assessment scale-cognitive subscale (ADAS-Cog) for the Portuguese patients with mild cognitive impairment and Alzheimer's disease. Clin Neuropsychol. 2018;32:46-59.

50. Tombaugh TN, Kozak J, Rees L. Normative data stratified by age and education for two measures of verbal fluency: FAS and animal naming. Arch Clin Neuropsychol. 1999;14:167-77.

51. Janelidze S, Mattsson N, Palmqvist S, et al. Plasma P-tau181 in Alzheimer's disease: relationship to other biomarkers, differential diagnosis, neuropathology and longitudinal progression to Alzheimer's dementia. Nat Med 2020;In Press.

52. Nakamura A, Kaneko N, Villemagne VL, et al. High performance plasma amyloid-beta biomarkers for Alzheimer's disease. Nature. 2018;554:249-54.

53. Schindler SE, Bollinger JG, Ovod V, et al. High-precision plasma beta-amyloid 42/40 predicts current and future brain amyloidosis. Neurology. 2019;93: e1647-59.

54. Hansson O, Janelidze S, Hall S, et al. Blood-based NfL: a biomarker for differential diagnosis of parkinsonian disorder. Neurology. 2017;88:930-7.

55. Preische O, Schultz SA, Apel A, et al. Serum neurofilament dynamics predicts neurodegeneration and clinical progression in presymptomatic Alzheimer's disease. Nat Med. 2019;25:277-83.

\section{Publisher's Note}

Springer Nature remains neutral with regard to jurisdictional claims in published maps and institutional affiliations.

Ready to submit your research? Choose BMC and benefit from:

- fast, convenient online submission

- thorough peer review by experienced researchers in your field

- rapid publication on acceptance

- support for research data, including large and complex data types

- gold Open Access which fosters wider collaboration and increased citations

- maximum visibility for your research: over $100 \mathrm{M}$ website views per year

At BMC, research is always in progress.

Learn more biomedcentral.com/submissions 